\title{
REPORTS OF NOTE
}

\section{Hierarchical Structures in Biology as a Guide for New Materials Technology}

1994

A recent discovery may lead to a better understanding of the structures of biomaterials that may result in the development of new synthetics with applications in the areas of aeronautics, biomedical research, and telecommunications. A new report from a committee of the National Research Council has presented the idea that current biomaterials may offer blueprints for new synthetics. Natural materials can provide lessons that will result in the development of new classes of synthetic materials.

Biomaterials have a system of hierarchical structures, which are formed when their individual units are combined to create larger and larger subunits. The report states that "learning to design and make synthetic composite materials that are structurally similar to mollusk shells could lead to stronger, stiffer, and more fracture-resistant materials for use in aircraft and spacecraft." The continued study of biomaterials to test their attributes amid different surroundings would be useful. Teams of scientists and engineers will design and produce new synthetic materials with complexities similar to biomaterials. The researchers conclude that the academic and industrial sectors should join them by implementing educational programs based on the systems' concepts and results.

National Academy Press

2101 Constitution Avenue, NW

Washington, DC 20055

$\$ 38.00$

\section{Equity and Equality in Health}

\section{4}

The forty-fourth session of the World Health Organization's Regional Committee for Europe met in Copenhagen, September 12-16, 1994, to discuss equity and equality in health care. While some may question the need to debate the concept of equity and equality in health care when there are serious problems of ill health to address, the report states that these issues are vital in making effective policy decisions on the major health challenges that confront us today.

The committee's report outlines the issues of equity and equality in health care followed by an examination of the disparities in health care and in opportunities for promoting health among European nations. The committee addressed several questions about the health care systems such as: is it ethical? is it fair? can we let these situations continue? The report further discusses what can be done to improve the situation. In the end, it explores possible steps that might be taken to address the dilemmas that were presented in this report.

World Health Organization, Regional Office for Europe, Copenhagen, Denmark

\section{Careers in Clinical Research Obstacles and Opportunities}

1994

Concerns about the dwindling numbers of individuals choosing clinical research as a career path prompted the Institute of Medicine in 1991 to set up a 16-member committee to look into the matter. As part of its charge, the committee was asked to explore the issues and make specific recommendations to remedy the situation.

The 344-page report defines the problem in some detail. Specifcally, it explores how clinical research is conducted in the United States, tackles issues such as education, training, and research funding, and outlines why health professionals become discouraged and what can be done to change this. A series of recommendations are made in the report to the US National Institutes of Health and other federal agencies supporting clinical research, universities and academic medical centres, government, industry, foundations, and professional societies.
The appendices contain the reports of the various task forces on clinical research in dentistry, nursing, clinical psychology, and surgery.

\section{National Academy Press} $\$ 39.95$

\section{Research Strategies for Assessing Adverse Events Associated with Vaccines A Workshop Summary}

1994

This document provides a summary of the proceedings of a workshop held on the subject in Washington, DC, in December 1993. Workshop participants included academic researchers, members of vaccine safety advocacy groups, vaccine manufacturers, health-care providers and representatives of federally funded research institutions and government agencies.

The participants were asked not to enter into the specifics about particular vaccines or adverse events, but to consider in more general terms how questions related to such topics should be tackled in the future. A consensus of opinion was not sought, and any opinions stated in the report are those of individual participants.

The three main topics discussed in the report are case reports and passive surveillance systems, large-linked databases and clinical trials. Introductory comments on each topic by a selected individual are followed by group discussions. Some discussion is given in the report to topics such as unvaccinated controls, the need for more information on the background incidence rates of the adverse events, adverse events with long latency periods, adverse events that are multifactorial in etiology, and the need for more basic research to understand the mechanisms of the adverse events.

National Academy Press $\$ 49.95$

\section{Compiled by Jennifer K. Snow} and Diane Gershon 